\title{
INTERAÇÃO ENTRE IDADE DA MATRIZ E PESO DO OVO NO DESEMPENHO DE FRANGOS DE CORTE
}

\section{Interaction of breeder age and egg weight on performance of broilers}

\author{
MUERER, R.F.P. ${ }^{1}$; VALLE, F.L.P. ${ }^{2}$; SANTOS, S.A. ${ }^{2}$; ZANATTA, C.P. ${ }^{2 ;}$ DAHLKE, F. ${ }^{3}$; \\ MAIORKA, A. ${ }^{3}$; OLIVEIRA, E.G. ${ }^{3}$
}

${ }^{1}$ Mestrando do Curso de Pós-Graduação em Ciências Veterinárias - UFPR
2 Graduandos do Curso de Zootecnia - UFPR
${ }^{3}$ Professores Adjuntos do Departamento de Zootecnia - UFPR

Endereço para correspondência: Alex Maiorka - amaiorka@ufpr.br

\section{RESUMO}

Existem vários fatores que podem afetar o desempenho e rendimento dos frangos de corte, independentemente dos níveis nutricionais utilizados nas dietas, como a idade da matriz e o peso do ovo. Neste trabalho, foram avaliados os possíveis efeitos que a idade da matriz e o peso do ovo à incubação exercem sobre o desempenho zootécnico e rendimento de carcaça de frangos de corte. As aves foram distribuídas em um delineamento experimental inteiramente casualizado em esquema fatorial $3 \times 3$ (ovos provenientes de matrizes de 3 diferentes idades: 30, 48 e 60 semanas, e de 3 categorias de pesos: leves, médios e pesados). As variáveis analisadas foram consumo de ração, ganho de peso e conversão alimentar aos 7, 21, 35 e 42 dias e rendimento de carcaça (rendimento de peito, pernas e gordura abdominal) aos 42 dias de idade. Não houve interação significativa entre os fatores idade da matriz e peso do ovo. Frangos provenientes de matrizes jovens (30 semanas de idade) apresentaram piores resultados de consumo de ração, ganho de peso e conversão alimentar à primeira semana de idade, sendo que este efeito não se mantém até a idade de abate. Houve maior consumo de ração de frangos provenientes de ovos pesados aos 7 dias de idade. Conclui-se que não existe interação entre idade da matriz e peso dos ovos sobre desempenho zootécnico e rendimento de carcaça em frangos de corte.

Palavras-chave: fatorial, incubação, matrizes pesadas, rendimento de carcaça.

\section{ABSTRACT}

There are several factors that can affect the performance and efficiency of broiler, regardless of the levels used in nutritional diets, such as breeder age and egg weight. In this study, were evaluated the effects of breeder age and egg weight carry on the performance and carcass yield of broilers. The birds were allocated in a completely randomized design in a factorial $3 \times 3$ (eggs from different breeder ages: 30 weeks, 48 weeks and 60 weeks and 3 categories of weights: light, medium and heavy). The variables analyzed were feed intake, weight gain and feed conversion to 7, 21, 35 and 42 days and carcass yield (carcass, chest, legs and abdominal fat) to 42 days old. There was not breeder age interaction for egg weight in broilers. Broilers from young breeders (30 weeks), presented worst results to feed intake, weight gain and feed conversion in first week, but this effect was not maintained until the slaughter age. The feed intake was increased on broilers from light eggs to 7 days old. It was concluded that no exist interaction between breeder age and egg weight on performance and carcass yield on broilers.

Key words: broiler breeder, carcass yield, factorial, hatchery. 


\section{INTRODUÇÃO}

Vários são os fatores que podem afetar o desempenho de frangos de corte, como a genética, nutrição, sanidade, manejo e ambiência. Entretanto, fatores estabelecidos antes mesmo da incubação e da eclosão, como a idade das matrizes e o peso dos ovos, são preponderantes para o desenvolvimento inicial das aves, tendo conseqüências em todo 0 crescimento (VIEIRA \& POPHAL, 2001; PEDROSO, et al., 2005).

A idade da matriz é um fator fisiológico determinante no tamanho de pintainhos, o que resulta em uma constante preocupação em relação ao estabelecimento de programas de alimentação e adoção de técnicas de manejo adequadas (MAIORKA, 2002). Sabe-se também que a idade da matriz tem influência direta sobre a qualidade e composição do ovo, pois matrizes velhas produzem folículos maiores, gerando também ovos com maior percentagem de gema, além de maior concentração de proteínas e fosfolipídios (CARDOSO, et al., 2002). Estas diferenças na composição dos ovos são importantes principalmente no momento final da incubação, quando ocorrerá a transferência de nutrientes do saco vitelínico para o embrião, sendo um fator que pode estar relacionado ao melhor desenvolvimento destas aves (APPLEGATE \& LILBURN, 1996). Esse melhor desempenho das aves provenientes de matrizes mais velhas foi comprovado por MAIORKA et al. (2003), que concluíram que frangos provenientes de matrizes velhas apresentaram maior consumo de ração e ganho de peso até os 42 dias de idade. Da mesma forma, PEEBLES et al. (1999), observaram influência da idade da matriz sobre rendimento de carcaça e rendimento de peito aos 43 dias de idade, sendo que as aves provenientes de matrizes mais velhas apresentaram os melhores resultados. Entretanto, também trabalhando com rendimento de carcaça de frangos de corte, DALANEZI et al. (2005) não observaram influência da idade da matriz sobre o rendimento de carcaça e pernas.

O peso dos ovos está diretamente relacionado com o peso ao nascimento de pintainhos de corte, sendo um fator que afeta o desempenho animal e pode provavelmente influenciar o peso das aves na idade de abate. Comprovando essa hipótese, PROUDFOOT et al. (1982) estudando a influência do peso do ovo sobre o peso de abate em frangos de corte, encontraram uma relação linear positiva, sendo que para cada grama a mais no peso do ovo, houve um ganho adicional de 10,7 gramas no peso de das aves no abate. Para PINCHASOV (1991), essa correlação positiva entre peso dos ovos e ganho de peso em frangos de corte existe, porém, esta correlação decresce com o aumento da idade.

Desta forma, este trabalho teve como objetivo avaliar a interação entre idade da matriz e o peso dos ovos sobre o desempenho zootécnico e rendimento de carcaça dos frangos de corte.

\section{MATERIAL E MÉTODOS}

Foram utilizados ovos embrionados da linhagem híbrida comercial Ross, provenientes de matrizes de 30,48 e 60 semanas de idade. Os ovos foram coletados no mesmo dia e receberam o mesmo manejo desde a sua coleta à chegada ao incubatório. Da produção total de cada lote (idade de matriz), foram pesadas amostras de $10 \%$ dos ovos coletados no mesmo dia, para obtenção do peso médio e desvio-padrão dos ovos, para cada idade de matriz. A partir destes valores, foram determinadas as faixas de peso dos ovos que foram classificados como ovos de peso leve, ovos de peso médio e ovos de peso pesado. Os tratamentos (idade das matrizes e peso dos ovos) e o peso inicial dos pintainhos com 
um dia de idade, são apresentados na Tabela 1.

Tabela 1 - Descrição dos tratamentos.

\begin{tabular}{cccc}
\hline $\begin{array}{c}\text { Trata- } \\
\text { mento }\end{array}$ & $\begin{array}{c}\text { Idade da } \\
\text { Matriz } \\
\text { (semanas) }\end{array}$ & $\begin{array}{c}\text { Classifica- } \\
\text { ção dos } \\
\text { Ovos }\end{array}$ & $\begin{array}{c}\text { Peso dos } \\
\text { Ovos } \\
\text { (gramas) }\end{array}$ \\
\hline T1 & 30 & Leves & $47,8-55,0$ \\
T2 & 48 & Médios & $55,1-62,3$ \\
T3 & 60 & Pesados & $62,4-69,6$ \\
T4 & 30 & Leves & $53,0-62,4$ \\
T5 & 48 & Médios & $62,5-71,9$ \\
T6 & 60 & Pesados & $72,0-81,4$ \\
T7 & 30 & Leves & $54,3-64,3$ \\
T8 & 48 & Médios & $64,4-74,4$ \\
T9 & 60 & Pesados & $75,5-85,5$ \\
\hline
\end{tabular}

Após a pesagem e classificação, os ovos foram identificados e acomodados em bandejas previamente identificadas $e$ incubados em incubadoras industriais Petersime $\AA^{\circledR}$, com temperaturas e umidades controladas em $37,5^{\circ} \mathrm{C}$ e $65 \%$, respectivamente. Aos 18 dias, os ovos foram transferidos para os nascedouros onde ficaram até 0 nascimento. Ao nascimento, realizou-se a sexagem através das penas das asas, onde foram coletados aleatoriamente 120 pintainhos machos de cada tratamento, totalizando 1080 aves. O delineamento experimental utilizado foi o inteiramente casualizado em esquema fatorial $3 \times 3$ ( 3 idades de matriz e 3 pesos de ovos) com 9 tratamentos e 6 repetições contendo 20 aves cada.

As aves foram alojadas em galpão experimental dividido em boxes e submetidas às mesmas condições ambientais (luz contínua de 24 horas de luz até os 14 dias de idade, ração isonutritiva à base de milho e farelo de soja e água $a d$ libitum durante todo 0 período experimental). As variáveis analisadas foram desempenho zootécnico (consumo de ração, ganho de peso e conversão alimentar), medido aos 7, 21, 35 e 42 dias, e rendimento de carcaça (rendimento de peito, pernas e gordura abdominal) aos 42 dias de idade. As médias obtidas foram submetidas à análise de variância, sendo comparadas pelo teste Tukey $(P<0,05)$.

\section{RESULTADOS E DISCUSSÃO}

Os resultados observados para consumo de ração aos 7, 21, 35 e 42 dias de idade de frangos provenientes de diferentes idades de matrizes e categorias de peso estão apresentados na Tabela 2. Não houve interação significativa $(P>0,05)$ entre os fatores idade da matriz e peso do ovo para a variável estudada. Analisandose os fatores principais, observa-se que os frangos oriundos de matrizes jovens (30 semanas de idade) tiveram um menor consumo de ração quando comparados aos frangos oriundos de matrizes velhas ou de idade intermediária (60 e 48 semanas de idade, respectivamente). Ao avaliar 0 efeito peso dos ovos, verificou-se que frangos provenientes de ovos pesados tiveram maior consumo de ração aos $7 \mathrm{e}$ 42 dias de idade.

Resultados semelhantes foram obtidos por DALANEZI et al. (2005), que também obtiveram maior consumo de ração para frangos oriundos de matrizes mais velhas, na fase inicial. Como frangos provenientes de matrizes mais velhas ou de ovos mais pesados tendem a ser maiores, é plausível supor que suas exigências sejam maiores, pois as exigências líquidas de energia e proteína aumentam à medida que aumenta o peso vivo do animal, levando ao maior consumo de ração para atender a essa exigência, além da maior capacidade física de ingestão.

As médias observadas para ganho de peso aos 7, 21, 35 e 42 dias de idade de frangos provenientes de ovos de diferentes categorias de peso e idade de matriz estão apresentadas na Tabela 3.

Frangos oriundos de matrizes de 30 semanas tiveram menor ganho de peso na 
Tabela 2 - Consumo de ração de frangos de corte oriundos de matrizes de 30, 48 e 60 semanas de idade, de ovos leves, médios e pesados aos 7, 21, 35 e 42 dias de idade.

\begin{tabular}{|c|c|c|c|c|}
\hline & \multicolumn{4}{|c|}{ Consumo de Ração (g/ave) } \\
\hline & $1-7$ dias & $1-21$ dias & $1-35$ dias & $1-42$ dias \\
\hline & \multicolumn{4}{|c|}{ Idade das Matrizes } \\
\hline 30 Semanas & $138,0 \mathrm{~b}$ & 1101,7 & 2915,4 & 4535,0 \\
\hline 48 Semanas & $154,8 \mathrm{a}$ & 1157,2 & 2968,2 & 4602,6 \\
\hline \multirow[t]{2}{*}{60 Semanas } & $151,1 \mathrm{a}$ & 1140,6 & 2956,6 & 4589,1 \\
\hline & \multicolumn{4}{|c|}{ Peso dos Ovos } \\
\hline Ovos Leves & $145,2 \mathrm{~b}$ & 1113,0 & 2914,4 & $4527,9 \mathrm{~b}$ \\
\hline Ovos Médios & $143,9 \mathrm{~b}$ & 1107,6 & 2911,6 & $4535,2 \mathrm{~b}$ \\
\hline \multirow[t]{2}{*}{ Ovos Pesados } & $154,8 \mathrm{a}$ & 1179,0 & 3014,2 & 4663,7 a \\
\hline & \multicolumn{4}{|c|}{ Probabilidade } \\
\hline Idade (I) & 0,0003 & 0,2001 & 0,6050 & 0,3189 \\
\hline Peso $(\mathrm{P})$ & 0,0159 & 0,0583 & 0,1167 & 0,0082 \\
\hline$(\mathrm{I} \times \mathrm{P})$ & 0,8730 & 0,7776 & 0,6853 & 0,6465 \\
\hline CV $(\%)$ & 9,52 & 8,53 & 5,59 & 3,27 \\
\hline
\end{tabular}

Médias seguidas de letras diferentes (a) na mesma coluna, dentro de cada fator, diferem entre si pelo Teste de Tukey (5\%).

Tabela 3 - Ganho de peso de frangos de corte oriundos de matrizes de 30, 48 e 60 semanas de idade, de ovos leves, médios e pesados aos 7, 21, 35 e 42 dias de idade.

\begin{tabular}{ccccc}
\hline & \multicolumn{4}{c}{ Ganho de Peso (g/ave) } \\
\hline & $1-7$ dias & 1-21 dias & 1 -35 dias & $1-42$ dias \\
\cline { 2 - 5 } & \multicolumn{4}{c}{ Idade das Matrizes } \\
\hline 30 Semanas & $100,2 \mathrm{~b}$ & 753,8 & 1985,4 & 2694,7 \\
48 Semanas & $109,3 \mathrm{a}$ & 783,7 & 2008,3 & 2711,3 \\
60 Semanas & $113,3 \mathrm{a}$ & 773,6 & 2010,6 & 2723,2 \\
\hline & & \multicolumn{3}{c}{ Peso dos Ovos } \\
\hline Ovos Leves & 104,9 & 765,9 & 1997,2 & 2694,7 \\
Ovos Médios & 106,3 & 752,0 & 1977,2 & 2673,0 \\
Ovos Pesados & 111,7 & 793,2 & 2029,1 & 2764,6 \\
\hline & & & \\
\hline Idade (I) & 0,0002 & 0,5337 & 0,7586 & 0,8438 \\
Peso (P) & 0,0606 & 0,3058 & 0,3929 & 0,1545 \\
(I x P) & 0,8730 & 0,8674 & 0,9070 & 0,6262 \\
CV (\%) & 9,68 & 10,17 & 5,36 & 5,37 \\
\hline
\end{tabular}

Médias seguidas de letras diferentes (a) na mesma coluna, dentro de cada fator, diferem entre si pelo Teste de Tukey (5\%). 
Tabela 4 - Conversão alimentar de frangos de corte oriundos de matrizes de 30, 48 e 60 semanas de idade, de ovos leves, médios e pesados aos 7, 21, 35 e 42 dias de idade.

\begin{tabular}{|c|c|c|c|c|}
\hline & \multicolumn{4}{|c|}{ Conversão Alimentar (g/g) } \\
\hline & 1-7 dias & 1-21 dias & 1-35 dias & $1-42$ dias \\
\hline & \multicolumn{4}{|c|}{ Idade das Matrizes } \\
\hline 30 Semanas & $1,3801 \mathrm{a}$ & 1,4623 & 1,4683 & 1,6864 \\
\hline 48 Semanas & $1,3203 b$ & 1,4808 & 1,4784 & 1,6996 \\
\hline \multirow[t]{2}{*}{60 Semanas } & $1,3358 b$ & 1,4783 & 1,4704 & 1,6865 \\
\hline & \multicolumn{4}{|c|}{ Peso dos Ovos } \\
\hline Ovos Leves & 1,3873 & 1,4575 & 1,4698 & 1,6837 \\
\hline Ovos Médios & 1,3557 & 1,4762 & 1,4718 & 1,7004 \\
\hline \multirow[t]{2}{*}{ Ovos Pesados } & 1,3931 & 1,4885 & 1,4856 & 1,6884 \\
\hline & \multicolumn{4}{|c|}{ Probabilidade } \\
\hline Idade (I) & 0,0462 & 0,5611 & 0,4961 & 0,6789 \\
\hline Peso $(\mathrm{P})$ & 0,5030 & 0,2184 & 0,2215 & 0,6092 \\
\hline$(I \times P)$ & 0,4439 & 0,9087 & 0,4487 & 0,5016 \\
\hline CV (\%) & 7,65 & 3,48 & 1,91 & 2,97 \\
\hline
\end{tabular}

Médias seguidas de letras diferentes (a) na mesma coluna, dentro de cada fator, diferem entre si pelo Teste de Tukey (5\%).

Tabela 5. Rendimento de carcaça (com pés e cabeça), peito, pernas e gordura abdominal de frangos de corte oriundos de matrizes de 30, 48 e 60 semanas de idade, de ovos leves, médios e pesados aos 42 dias de idade.

\begin{tabular}{ccccc}
\hline & \multicolumn{4}{c}{ Rendimentos (\%) } \\
\hline & Carcaça & Peito & Pernas & Gordura Abdominal \\
\cline { 2 - 5 } 30 Semanas & \multicolumn{4}{c}{ Idade das Matrizes } \\
48 Semanas & 83,65 & 31,79 & 26,48 & 1,80 \\
60 Semanas & 84,00 & 30,78 & 25,84 & 1,99 \\
\hline & 84,70 & 31,46 & 26,03 & 2,05 \\
\hline Ovos Leves & 84,35 & \multicolumn{4}{c}{ Peso dos Ovos } \\
Ovos Médios & 84,07 & 31,79 & 25,67 & 1,85 \\
Ovos Pesados & 83,93 & 31,00 & 26,28 & 1,99 \\
\hline & & 26,39 & 2,00 \\
\hline Idade (I) & 0,6770 & 0,0782 & 0,2314 & 0,1223 \\
Peso (P) & 0,9388 & 0,1997 & 0,1327 & 0,3945 \\
(I x P) & 0,9273 & 0,9446 & 0,8785 & 0,0967 \\
CV (\%) & 4,02 & 4,27 & 4,33 & 20,33 \\
\hline
\end{tabular}


primeira semana. Este resultado pode ser reflexo da menor ingestão de ração dos frangos de corte provenientes deste grupo. Dados semelhantes foram encontrado por SINCLAIR et al. (1989), GLADYS et al. (2000), MENDES et al. (2001), onde frangos oriundos de matrizes de 30 semanas também tiveram menor ganho de peso apenas na primeira semana, não havendo diferenças entre os tratamentos $(P>0,05)$ nas demais idades avaliadas. Esta ausência de efeitos no período restante, provavelmente se deve ao fato das aves provenientes de ovos mais leves ou de matrizes mais jovens, aumentarem seu consumo, resultando em um peso vivo proporcionalmente maior em relação às outras aves.

As médias observadas para conversão alimentar aos 7, 21, 35 e 42 dias de idade de frangos estão apresentadas na Tabela 4. Os frangos de corte provenientes de matrizes de 30 semanas de idade tiveram pior conversão alimentar quando comparados aos frangos de corte oriundos de matrizes de 48 e 60 semanas de idade. Resultados semelhantes foram encontrados por PEEBLES et al. (1999), onde frangos de corte provenientes de matrizes mais novas obtiveram os piores resultados de conversão alimentar. Os resultados de desempenho ao final dos 42 dias concordam com BARRETO (1999) e CANÇADO et al. (1995), que verificaram que a idade da matriz e o peso dos ovos não afetaram o desempenho zootécnico das aves na idade de abate.

Os resultados de rendimento de carcaça (rendimento de carcaça, peito, pernas e gordura abdominal) estão apresentados na Tabela 5.

Não houve interação significativa entre os fatores idade da matriz e peso dos ovos para nenhuma das variáveis estudadas. Da mesma forma, ROCHA et al. (2007), não encontraram interação significativa entre os mesmos fatores analisados. Quando analisado os fatores principais, também não foi observada diferença para rendimentos de carcaça, peito, pernas e gordura abdominal. Estes resultados corroboram com VIEIRA \& MORAN (1998), que concluíram que a idade da matriz parece não ter efeitos no rendimento de carcaça, ocasionalmente por também não ter ocorrido diferenças no peso final das aves.

\section{CONCLUSÕES}

Nas condições em que foi conduzido este trabalho, conclui-se que não existe interação entre idade de matriz e peso dos ovos sobre as características de desempenho e de rendimento da carcaça de frangos de corte.

A idade da matriz e o peso do ovo é fator preponderante no desenvolvimento de frangos de corte somente na primeira semana de idade.

O rendimento de carcaça e gordura abdominal de frangos de corte à idade de abate não são alterados pela idade da matriz ou peso dos ovos.

\section{REFERÊNCIAS}

APPLEGATE, T.; LILBURN M.S. Characteristics of changes in yolk sac and liver lipids during embryonic and early posthatch development of turkey poults. Poultry Science, v.75, p.478-483, 1996.

BARRETO, S.L.T. et al. Níveis de proteína e de vitamina e para matrizes de frango de corte e seus efeitos sobre 0 desempenho das matrizes, composição do ovo e desempenho da progênie. Arquivo Brasileiro de Medicina Veterinária e Zootecnia, v.51, n.2, 1999.

CANÇADO, S. V. et al. Ácido linoléico na alimentação de matriz pesada. Efeitos sobre: peso do ovo, incubação, relação peso da gema/peso do ovo e qualidade dos pintos. In: Conferência APINCO de Ciência Tecnologia Avícolas, 1995, Campinas. Anais... FACTA: Campinas, 1995. p.127-128.

CARDOSO, J.P. et al. Efeito da idade da matriz e peso dos ovos, sobre os componentes do ovo em frangos de corte. Revista Brasileira de Ciência Avícola, Campinas, v.4, p.16, 2002. 
DALANEZI, J.A. et al. Efeito da idade da matriz sobre o desempenho e rendimento de carcaça de frangos de corte. Arquivo Brasileiro de Medicina Veterinária e Zootecnia, v.57, n.2, 2005.

GLADYS, G.E. et al. Effect of breeder flock age and diet density on broiler performance. Poultry Science, v.79, p.123, 2000.

MAIORKA, A. et al. Idade da matriz e qualidade do pintainho. In: MACARI, M.; GONZALES, E. Manejo da Incubação. $2^{\mathrm{a}}$ edição. Campinas: FACTA, 2003, v.1, p. 361-377.

MAIORKA, A. Efeito da idade da matriz e do agente trófico (glutamina) sobre 0 desenvolvimento da mucosa intestinal e atividade enzimática do pâncreas de pintos de corte na primeira semana. Tese (Doutorado em Zootecnia), Universidade Estadual Paulista, Jaboticabal, 2002, 100p.

MENDES, A.A. et al. Avaliação do rendimento e qualidade da carne de peito em frangos de corte criados com diferentes densidades e níveis de energia na dieta. Revista Brasileira de Ciência Avícola, v.3, p.38, 2001.

PEDROSO, A.A. et al. Desempenho e biometria de órgãos digestórios de frangos provenientes de matrizes jovens após diferentes intervalos de alojamento. Revista Brasileira de Ciência Avícola, Campinas, v.7, p.5, 2005.
PEEBLES, E.D. et al. Effects of breeder age and dietary fat on subsequent broiler performance. Poultry Science, v.78, p.512-515, 1999.

PINCHASOV, Y. Relationship between the weight of hatching eggs and subsequent early performance of broiler chicks. British Poultry Science, v.32, p.109115, 1991.

PROUDFOOT, F.G. et al. Effect old hatching egg size from semi-dwarf and normal maternal meat parent genotypes on the performance of broiler chickens. Poultry Science, v.61, p.655-660, 1982.

ROCHA, J.S.R.. et al. Efeito da idade da matriz e do tamanho do ovo sobre o rendimento de abate do frango de corte. In: Conferência APINCO de Ciência e Tecnologia Avícolas, 2007, Santos. Anais... FACTA: Campinas, 2007. p.8.

SINCLAIR, R. W. et al. The effects of parentage post hatch treatment on broiler performance. Poultry Science, v.69, p.526-534, 1989.

VIEIRA, S.L.; MORAN Jr., E.T. Eggs and chicks from broiler breeders of extremely different age. Journal of Applied Poutry Research, v.7, p.372376, 1998.

VIEIRA, S.L.; POPHAL, S. Eclodibilidade de ovos oriundos de matrizes com extremos em idades e de pesos distintos. Revista Brasileira de Ciência Avícola, v.3, p.5, 2001. 